Grammaire et enseignement du français langue étrangère et seconde - Permanences et ruptures du XVIe au milieu du XXe siècle (II)

\title{
Remonter à l'origine des règles pour dénouer la complexité grammaticale
}

\section{Aurelio Principato}

\section{(2) OpenEdition \\ Journals}

\section{Édition électronique}

URL : https://journals.openedition.org/dhfles/3632

DOI : $10.4000 /$ dhfles.3632

ISSN : 2221-4038

\section{Éditeur}

Société Internationale pour l'Histoire du Français Langue Étrangère ou Seconde

Édition imprimée

Date de publication : 1 juin 2014

Pagination : 181-193

ISSN : 0992-7654

Référence électronique

Aurelio Principato, "Remonter à l'origine des règles pour dénouer la complexité grammaticale », Documents pour l'histoire du français langue étrangère ou seconde [En ligne], 52 | 2014, mis en ligne le 02 août 2016, consulté le 28 mai 2021. URL : http://journals.openedition.org/dhfles/3632 ; DOI : https:// doi.org/10.4000/dhfles.3632

Ce document a été généré automatiquement le 28 mai 2021.

(c) SIHFLES 


\title{
Remonter à l'origine des règles pour dénouer la complexité grammaticale
}

\author{
Aurelio Principato
}

1 Mon exposé vise la notion de simplification pédagogique, qui est parmi les thèmes en discussion dans ce colloque. Cette occasion a sollicité mes réflexions ultérieures concernant les principes qui m'ont inspiré dans la préparation, avec Francesco Paolo Alessandro Madonia, de la Grammatica della lingua francese, publiée chez Carocci en 2011.

2 En effet, cette grammaire étant adressée à un public universitaire, elle se veut affranchie, en principe, des contraintes d'une facilitation qui tienne compte de l'âge des apprenants et de leur approche "précoce» des complexités grammaticales d'une langue étrangère. Nous avons conçu un apprentissage réfléchi pour un adulte, qui ne devrait pas susciter cette demande. Mais le principe de simplification ne reste pas moins valable : à condition, certes, de l'entendre dans un autre sens.

Le critère que nous avons adopté devrait permettre à l'élève de s'orienter parmi les règles et leurs exceptions: un ordre explicatif, dont l'apprenant puisse se servir de boussole dans la jungle constituée par des listes de paradigmes ou d'irrégularités dont la présentation traditionnelle ne laisse pas trop saisir la « logique ».

C'est une idée semblable que le XVIII ${ }^{\mathrm{e}}$ siècle avait poursuivie, notamment en ce qui concerne les manuels destinés... aux dames, et non seulement de grammaire. Il s'agit de la reprendre en quelque sorte, aujourd'hui, avec l'apport des acquisitions qui se sont accumulées, grâce à l'énorme développement des sciences philologiques survenu depuis le XIX ${ }^{e}$ siècle.

5 Pour appliquer aujourd'hui cette forme de simplification, il ne faut pas faire économie d'explications. D'autant plus que nous nous adressons à un public adulte. Même sans entrer dans les théories cognitives, on constate d'ailleurs que, lorsque la présentation d'une norme ou d'une exception est accompagnée d'une explication qui renseigne quant à l'origine de telle particularité morphologique ou de telle contrainte syntaxique et ainsi de suite, l'objet en question se fixe beaucoup plus facilement dans la mémoire. 
6 Profitant donc de l'occasion que m'offrait ce colloque pour confronter cette stratégie d'apprentissage à l'histoire de l'enseignement du français, j'ai cherché comme objet de comparaison une grammaire qui, sans être vraiment ancienne, soit censée témoigner d'un souci de simplification telle qu'on l'entend normalement.

7 J'ai choisi dans ce but la Grammatica francese de Giuseppe Presenzini, publiée pour la première fois en 1946, et rééditée jusqu'à l'époque où, en Italie, l'avènement en force de la méthode directe a totalement changé la donne et rendu la production grammaticale ultérieure largement incomparable avec notre idée d'un apprentissage réfléchi des règles. L'édition que j'ai consultée est la $6^{\mathrm{e}}$ (1957, Milano : Garzanti), dans l'exemplaire qui avait servi à mon vieil ami Giovanni Saverio Santangelo, car elle était adoptée la même année aux Scuole Medie de Palerme et, comme nous nous sommes retrouvés depuis sur les mêmes bancs de lycée, elle aurait pu être ma grammaire aussi, si j'avais fait du français lors de mes études inférieures.

Conformément à la tradition des grammaires à usage pratique, Presenzini utilise les dialogues comme une forme de méthode directe ${ }^{1}$, et exploite assez leur utilité en fonction contrastive. Sa grammaire comporte en annexe des listes de synonymes, de proverbes, de phrases types, qui s'adaptent à l'approche foncièrement onomasiologique des grammaires pour une langue seconde, dans la mesure où elles prennent la phraséologie, italienne dans notre cas, comme point de départ pour montrer en quoi elle correspond à la française. Cela rend des services notamment en ce qui concerne les adverbes, les prépositions, les conjonctions.

Nous en avons tenu compte aussi dans les chapitres consacrés aux parties invariables du discours et à la phrase. Mais, outre la destination différente et la présence d'exercices, c'est au niveau de l'explication grammaticale qu'il faut juger des éléments qui distinguent la grammaire en question de la nôtre.

Or, dans la mesure où Presenzini s'efforce de suggérer la logique de certaines règles ou de suggérer des critères mnémotechniques, son attitude de n'est pas si éloignée de celle qui a inspiré notre grammaire.

11 Dans sa préface, outre à mettre en valeur certains aspects (l'abondance des exercices notamment), l'auteur tient à souligner la place qu'il a accordée à la phonétique et à la prononciation, ainsi qu'à l'organisation qu'il adopte dans l'exposition des règles. Il oppose ces propriétés de sa grammaire au désordre engendré, dans d'autres manuels pratiques, par le morcellement de la matière en «leçons" qui suivent l'ordre séquentiel de l'apprentissage.

12 Presenzini annonce aussi son intention de faciliter l'étude de la conjugaison verbale par l'extraction d'un noyau de base, constitué par des formes («voci ») fondamentales, d'où naissent les formes « dérivées ».

13 Il s'agit de critères assez proches des nôtres. Observons-les dans l'ordre.

\section{L'organisation descriptive}

14 Alors que nous avons la chance de pouvoir orienter notre public adulte par des renvois d'un chapitre à l'autre, Presenzini est obligé de se soumettre à la progression pédagogique. Pour rester cohérent avec son souci d'assurer néanmoins une exposition méthodique, il parsème alors son texte d'encarts qui respectent l'ordre d'apprentissage proposé. 
Afin de ne pas créer d'obstacle à l'entrée du lecteur dans la matière, nous avons également opté pour sa division traditionnelle en "parties du discours", que nous avons fait précéder de deux chapitres consacrés respectivement à «Phonétique et phonologie » et "Graphématique et orthographe ». Le reste est plus conforme au plan traditionnel que présentent nombre de grammaires pour étrangers depuis au moins le XVIII ${ }^{\mathrm{e}}$ siècle. Suivent ainsi "L'article », «Le nom », "L'adjectif », « Le pronom », « Le verbe », «L'adverbe », «La préposition», «La conjonction». Les deux derniers chapitres («La phrase simple» et «Syntaxe de la phrase complexe») se chargent d'établir la taxinomie des différentes réalisations syntaxiques en s'appuyant sur les jalons présentés tour à tour dans les chapitres précédents à propos des différentes parties du discours. générales de l'évolution $\mathrm{du}$ français, pour servir de repère aux digressions diachroniques contenues dans les chapitres et dont je vais plus loin préciser la nature. Mon co-auteur Madonia s'est chargé de la rédaction d'un tiers de ces chapitres, trois autres collaborateurs (Simona Pollicino, Pietro Cancelliere, Paolo Frassi) se sont partagé les autres, alors que j'ai réservé pour moi l'introduction historique, les encadrés diachroniques et le chapitre «Graphématique et orthographe ». La révision de l'ensemble a été menée collectivement, avec la collaboration très importante de Valeria Di Stefano et de Valerio Emanuele.

20 Nous avons rattaché la prononciation au chapitre "Graphématique et orthographe » pour laisser la plus grande autonomie à la «Phonétique et phonologie ». Cette distribution,inhabituelle dans les grammaires, jointe à la distinction entre l'orthographe et la graphématique, vient du fait que, ne serait-ce que par son étymologie, l'orthographe renvoie en effet à la norme, alors que nous voulons, au contraire, illustrer les différentes contraintes qui ont engendré le système graphique dans son état actuel.

21 Je trouve intéressant, sur ce point, que Presenzini crée un lien entre deux phénomènes d'ordre graphématique, en juxtaposant le tréma et le H entre voyelles (Presenzini : 13), lorsqu'il est question de prononciation. De même, au moment d'illustrer la prononciation de mots tels que cueillir, Presenzini la justifie par l'inversion du digramme eu dans le but de permettre au C de garder le son dur ${ }^{2}$. 


\section{Le choix de la taxinomie italienne} nous sommes posé d'emblée le problème de la réelle correspondance de ce modèle avec l'objet à traiter. Ce qui nous a fait trancher en faveur de l'adoption de la nomenclature italienne a l'avantage de pouvoir nous rattacher par là à la formation scolaire de notre public.

Dans la conviction qu'une terminologie et un ordre plus traditionnels répondent à l'exigence de clarté que l'apprenant doit ressentir en abordant notre grammaire, nous avons préféré créer un cadre à l'intérieur duquel nous avons pu signaler les nouvelles perspectives linguistiques, en y renvoyant de façon concise à l'occasion. Cela a comporté l'adoption d'une taxinomie et d'une terminologie italiennes.

Jusqu'à quel point ce choix a-t-il été légitime? On pourrait, en effet, considérer que chaque langue est un objet particulier qui demande son propre métalangage descriptif.

Dès les premiers moments, on a pris conscience que les catégories grammaticales françaises traditionnelles ne correspondent pas aux catégories italiennes courantes. Pour citer un exemple que j'ai récemment évoqué (Principato 2012), la nomenclature des complétives est différente en français et en italien à cause du statut pro-drop de l'italien, qui permet d'aligner sur les complétives sujet les séquences de verbes impersonnels, alors que cela est impossible en français à cause de la présence d'un sujet grammatical «vide». Ainsi deux propositions qui se ressemblent comme deux sœurs jumelles et qui ont exactement la même signification en italien et en français :

mi sembra importante che tu gli parli

il me semble important que tu lui parles

seront classées respectivement comme subjective et terme complétif du sujet, car la taxinomie française réserve le nom de complétive sujet aux seules propositions qui occupent la tête de la phrase :

que tu lui parles me semble important

Parfois, Presenzini reste pris au piège de cette difficulté. Il traite, par exemple, le gerundio comme un mode indéfini à part entière, tel qu'il se présente en italien. Il distingue ainsi la « forme simple du gérondif » de celle qui est construite avec en. C'est le choix le plus naturel dès qu'on adopte, comme perspective, trois traductions possibles du « gerundio » italien (Presenzini : 291-293).

Mais, en italien, le gerundio a une morphologie propre, alors que le gérondif français peut être classé comme une construction spéciale du participe présent. Nous avons donc évité d'introduire cette notion dans la morphologie du verbe mais, lorsqu'il est question des modes, des temps et, notamment, des emplois, de signaler plutôt qu'il correspond au participe présent français.

8 La différence qui existe, d'une langue à l'autre, entre différents systèmes descriptifs recoupe celle qui oppose le métalangage de la linguistique à celui des grammairiens. Cela apparaît encore mieux, dans la seconde partie de la grammaire de Presenzini, lorsqu'il opère la transition de l'italien au français: sans avoir préparé d'abord l'étudiant, il se trouve alors à utiliser dans le sens français la notion d'« attribut » (ibid. : 350), qu'un Italien pourrait confondre avec la fonction logique de l'adjectif.

Écartant donc l'idée d'adapter à nos buts une grammaire française déjà existante, bien qu'il y en ait d'excellentes, et penchant plutôt pour le modèle taxinomique italien, nous 
est vrai que, dans la plupart des cas, il suffit de se rapporter à la traduction courante des outils terminologiques. Il a donc été aisé d'avoir recours à legamento pour « liaison » et à cediglia pour « cédille » - ce dernier mot d'ailleurs tirant à son tour son origine de l'espagnol - malgré l'absence de la liaison et de la cédille dans la langue italienne.

On pourrait qualifier de rétrograde le fait que nous ayons gardé la catégorie d'adjectifs déterminatifs au détriment de celle de pré-déterminants, qui recouvre également l'article. Mais cela n'est pas si évident si l'on considère la possibilité qu'a l'italien de cumuler l'article et le possessif, alors qu'en français la possibilité de cumul est plus restreinte.

Le choix de publier cette grammaire en langue italienne, choix déterminé d'ailleurs par des raisons éditoriales, a été donc moins important que d'autres options. La décision qui a marqué davantage la structure de notre ouvrage a porté sur la distribution des matières et sur la terminologie adoptée. La dernière est en rapport avec la formation de nos premiers destinataires.

Alors qu'il nous a été possible de comparer à l'occasion les taxinomies et les points de vue italiens et français, qui s'avèrent souvent être divergents, au contraire, chez Presenzini, l'organisation de la matière se heurte souvent à l'absence de critères adéquats.

Pour expliquer la généralisation au singulier de la forme interrogative est-ce?, il a recours aux arguments du temps de Vaugelas, à savoir que sont-ce? et seront-ce? sont «désagréables à l'oreille» (Presenzini : 285)3. . De même, il qualifie correctement d'invariable l'adjectif grand dans grand'mère, etc. (ibid. : 342 et 346 ), mais ne s'exprime pas sur la présence de l'apostrophe, qui pourrait faire penser à l'élision.

Arrivons à la conjugaison verbale (ibid. : 180 suiv.). Toujours chez Presenzini, les verbes sont distribués sur 4 conjugaisons selon la distinction traditionnelle des terminaisons de l'infinitif. Afin de pouvoir attribuer un paradigme à la conjugaison des verbes en -oir et d'y insérer au moins quelques verbes, il est obligé d'indiquer comme réguliers devoir, recevoir et ceux de la même famille, quitte à signaler la présence d'une "radicale mobile ", qui tantôt reste inaltérée, tantôt se change en -oiv-, tantôt disparaît (je reçois) et à rassurer que, pour simplifier l'étude, l'élément ev sera pris comme faisant partie de la terminaison... On voit bien comment, loin de nous rassurer, le souci de simplification peut engendrer une complexité nouvelle.

Quant à nous, nous avons rapporté cette alternance, commune à beaucoup d'autres verbes, à l'ancienne diphtongaison de la voyelle tonique. Nous avons fait de même pour ce qui est de l'alternance $i / y$ et pour d'autres phénomènes morpho-graphiques. Autrement dit, nous remontons à la phonétique et à la graphématique, qui relie les fondements du système phonologique établis dans le premier chapitre et le système d'écriture qui en est issu, pour faire de l'évolution historique le fondement de la morphologie actuelle. La phonétique et la graphématique deviennent ainsi la matrice de la description qui sera consacrée, au cours des chapitres suivants, aux différentes parties du discours.

37 À titre d'exemple, un seul phénomène tel que l'ancienne vocalisation de $l$ en fin de syllabe est invoqué pour rendre compte de plusieurs phénomènes morphologiques et morphosyntaxiques: la forme des articles contractés au masculin singulier ainsi que celle des pronoms eux et ceux, la formation des pluriels en -ux, l'alternance en/au devant le nom de pays et de saison, celle de $-\boldsymbol{l}$ - et $-\mathfrak{u}$ - dans le paradigme des verbes 
falloir, valoir, vouloir, dissoudre et, comme corollaire, l'apparition/disparition du -l- dans celui de moudre.

Cette ancienne vocalisation du $l$ implosif est introduite au chapitre 2 ("Graphématique et orthographe ») au moment où sont présentés les pluriels en -ux. Son illustration comporte un complément indispensable d'histoire de la culture, qui est de rappeler des conventions manuscrites du moyen-âge ayant entrâné ultérieurement la confusion du -us qu'on prononçait et du $x$ qu'on lisait et, par là, l'insertion d'un -u- de plus. On n'a pas négligé de rendre compte, au passage, de l'insertion, plus tard, d'un -l- étymologique supplémentaire et de la résistance de celui-ci dans la graphie des noms propres (Renault, Foucault) : c'est l'occasion de rappeler aux étudiants que ce $l$ est muet !

L'information, encadrée à l'intérieur de la description graphématique, se projette par ce biais sur l'existence de formes particulières du pluriel, dont il sera question aux paragraphes traitant la formation du pluriel du chapitre consacré au « Nom ». Du coup, au moyen d'un rappel au phénomène décrit deux chapitres plus haut, la présence de cette forme de pluriel dans la morphologie française perdra tout caractère d'« exception ».

Pour évoquer d'autres cas présents dans la conjugaison des verbes, le même principe explicatif intervient pour rendre compte de la survivance du modèle jeter (il jette), à côté de l'orthographe d'autres verbes où le $e$ prend l'accent grave en position tonique lorsqu'il est suivi d'une syllabe avec $e$ caduc (il mène, elle achète). L'avènement des accents et de nouveaux signes diacritiques à l'époque humaniste, qui avait été signalé là où il est question de la coprésence de la notation graphématique ancienne fondée sur la combinaison des lettres, devient ainsi également le support qui justifie le traitement de la forme des noms et des adjectifs au féminin : tienne, muette, originelle d'un côté et, de l'autre, complète, concrète.

41 Pour revenir aux intentions qui nous ont animés, c'est la présentation de longues listes qu'on a voulu éviter, comme on en trouve, bien entendu, chez Presenzini, telles que les 20 exceptions relatives à la prononciation (Presenzini : 32-34) et les «irrégularités diverses" ou les "formes spéciales" concernant le féminin (ibid.: 77 et 83-84) et confiées uniquement à la mémoire de l'apprenant.

u encore, nous expliquons la prononciation particulière de femme en la mettant en relation avec l'ancienne nasalisation, et en l'homologuant par ce biais aux adverbes dérivés d'adjectifs en -ent (violemment, prudemment), qui ont été affectés ultérieurement par la dénasalisation.. Ou encore, pour ce qui est des noms d'agent en -eur,la formation du féminin en -euse est rapportée à la chute des consonnes finales, qui culmine au XVII ${ }^{\mathrm{e}}$ siècle.

43 Celle du genre est une question qui mérite un développement. On a toujours parlé de "formation du féminin », et par conséquent de transformation de -eux final en -euse, de -eau en -el(le), de $-f$ en -ve, et ainsi de suite. Presenzini ne manque pas de suivre cette habitude (ibid. : 72-74, 76-78) et, en plus, il sépare les terminaisons en -eux et celles en eur, tout en observant bien à propos que c'est l'origine française ou latine qui distingue ces dernières. Mais à son époque on faisait encore beaucoup de latin...

Il va sans dire que Presenzini avait commencé par parler d'un -e ajouté à la forme du masculin. Il faudra attendre encore une vingtaine d'années et que la primauté soit accordée à l'oral pour assumer que la marque du féminin est la consonne finale qui réapparaît à l'oral. On a continué pourtant à oublier que c'est la forme féminine qui est 
conservatrice, et que s'il y a transformation, c'est celle qui est intervenue historiquement dans la forme du masculin. Le temps me semble enfin venu de renverser là aussi les rôles de genre !

Nous avons offert quelques explications d'ordre historique relatives aussi à la variation sémantique. Cet aspect est présent surtout dans les chapitres lexicologiques et dans ceux qui concernent les invariables. Dans ce dernier cas, la diachronie s'avère très utile pour illustrer les phénomènes de conversion catégorielle auxquels ont été constamment soumises ces parties du discours, avec le changement sémantique qui a accompagné leur conversion: l'origine, par exemple, des adverbes pas, maintenant, soudain, pile, les prépositions sauf ou pendant.

Les lignes générales de l'influence des aspects culturels sur les choix orthographiques et sur les tendances à les normaliser ayant déjà été rappelées, il nous a suffi à l'occasion de rappeler brièvement l'influence de la morphologie latine.

\section{Présentation graphique de la variation}

L'insertion des informations diachroniques se rapporte ainsi au fonctionnement général du code et aux déviations par rapport à celui-ci. Avant Grevisse, la séparation était nette entre les grammaires destinées à l'enseignement de la langue d'un côté et, de l'autre, les « grammaires historiques » - ou plutôt les études spécialisées (Brunot et Bruneau 1969, Fournier 1998...). D’ailleurs, l'orientation normative dominante a largement encouragé une vision en quelque sorte atemporelle des règles, alors que les finalités d'ordre scolaire ou pratiques à poursuivre ne semblaient guère s'accorder avec la diachronie.

49 L'innovation que nous avons essayé d'apporter consiste aussi à nous démarquer de la séparation, toujours présente dans Grevisse et Goosse (2007), entre la description de l'usage et l'information diachronique. Cette dernière est entendue, dans notre grammaire, comme la clé d'explication des particularités grammaticales.

Les commentaires historiques, offrant une description aussi brève que possible du phénomène dont il est question, sont encadrés dans le texte, ainsi que d'autres concernant la variation diastratique, qui se distinguent des premiers par l'emploi des caractères italiques. Plutôt que sur les différents français, nous avons estimé que l'intérêt de notre public porte davantage sur les registres de langue. Ces encarts informent donc au besoin, d'une façon qui ne saurait être systématique, d'usages divers propres au registre familier, ou avisent sur l'emploi restreint de telle locution ou construction syntaxique. Ils s'efforcent en somme de mettre au courant l'apprenant italien de l'écart qui sépare la langue vivante de celle qui fait l'objet de l'apprentissage d'une langue étrangère, lorsqu'il s'exerce loin de la pratique quotidienne. Du côté des étudiants, ils visent à atténuer l'impression de distance qui peut se créer vis-à-vis du français standard, et à les ramener au sentiment d'une koïnè « jeune ».

D'ailleurs, ces encarts n'interrompent pas la continuité de la description grammaticale, c'est-à-dire que la possibilité reste d'une lecture qui en fasse l'économie.

52 Par ce moyen, les commentaires demeurent tout de même plus visibles que des notes en bas de page ou en marge. Une séparation excessive est ainsi évitée entre la description de l'état actuel de la langue et les aperçus qui montrent celui-ci comme étant le fruit d'un processus en diachronie et varié en synchronie. 

considérer une telle distinction graphique que comme un simple moyen pour accroître la lisibilité. L'expérience en classe nous a montré d'ailleurs que le fait même d'attirer l'attention sur l'explication diachronique pousse les élèves à consacrer à cet aspect autant d'attention qu'aux parties de texte non encadrées.

Pour conclure, le critère de simplification courant reste valable pour une grammaire scolaire, où il ne serait pas plus possible d'insérer des encadrés de ce genre que de présenter une exposition systématique de la phrase simple et de la phrase complexe. Mais on pourrait y ajouter le nouveau critère que je viens de décrire, à condition de chercher des principes de regroupement pour des phénomènes apparemment hétérogènes. À ce sujet, l'histoire de la langue pourrait offrir des suggestions, pour sortir du galimatias normatif et des aspérités mnémotechniques qui entravent souvent notre mission d'enseignants.

Par conséquent, tout en nous adressant à un public universitaire, nous espérons avoir procuré une grammaire qui puisse servir d'outil pour toute catégorie d'enseignants et pour la construction d'autres nouvelles, destinées à l'enseignement inférieur également.

\section{BIBLIOGRAPHIE}

BIDAUD, Françoise (2008). Nouvelle grammaire du français pour italophones. Torino : UTET. BRUNOT, Ferdinand \& BRUNEAU, Charles (1969 [1949]). Précis de grammaire historique de la langue française. Paris : Masson et Cie.

FOURNIER, Nathalie (1998). Grammaire du français classique. Paris : Belin.

GREVISSE, Maurice \& GOOSSE, André (2007 [1936]). Le Bon Usage. Grammaire française. Bruxelles : De Boeck.

MINERVA, Nadia, dir. (1998). Les dialogues dans les enseignements linguistiques : profil historique, Actes de la journée d'études organisée à Bologne le 2 mars 1996, édités avec l'aide d'Anna Maria Mandich et Carla Pellandra. Documents pour l'histoire du français langue étrangère ou seconde, 22.

PRESENZINI, Giuseppe. Grammatica francese (1957 [1946]). Milan : Garzanti.

PRINCIPATO, Aurelio (2012). « Le sujet dont on parle (notes sur les rôles accordés au pronom impersonnel)», Diachroniques, 2, 153-171.

RIEGEL, Martin \& PELLAT, Jean-Christophe \& RIOUL, René (2009 [1994]). Grammaire méthodique du français. Paris : P.U.F.

SCHENA, Leandro (1991). Étude descriptive de la phrase française, ses constituants, ses formes, ses transformations. Milan : Pubblicazioni dell'I.S.U. - Università Cattolica.

SERIANNI, Luca (1996 [1988]). Grammatica italiana. Italiano comune e lingua letteraria. Turin : UTET. 
VAUGELAS, Claude Favre de (2009 [1647]). Remarques sur la langue françoise. Édition critique avec introduction et notes par Z. Marzys. Genève : Droz.

\section{NOTES}

1. Voir, sur cet aspect, Minerva 1998.

2. En fait, c'est l'inversion contraire qui a eu lieu, ex. cuer $>$ cœur.

3. Cf. Vaugelas $2009: 360$.

\section{RÉSUMÉS}

La «simplification pédagogique » est-elle compatible avec un apprentissage réfléchi des règles, aidé par l'illustration du système grammatical où elles s'insèrent, ainsi que par leur origine historique?

En outre, au lieu de réduire ses contenus, une grammaire italienne de la langue française devrait viser à stimuler la comparaison entre les taxinomies grammaticales italienne et française.

Pour interroger les limites de l'approche traditionnelle à ce sujet, l'article analyse la grammaire de Presenzini, parue pour la première fois en 1946. Cette grammaire amorçait en effet la recherche de principes unificateurs qui expliquent les particularités et les exceptions phonographématiques et morphologiques du français.

Ces options sont à l'origine d'une nouvelle grammaire destinée à un public universitaire italien (F. P. A. Madonia et A. Principato, Grammatica della lingua francese, Roma, Carocci, 2011).

Is 'pedagogic simplification' compatible with developing a reflective approach to the grammatical rules, by introducing them within the frame of the grammar system and by explaining their historical origins?

Away from shortening its contents, an Italian grammar of French language should stimulate the reflection on different categories employed for French or Italian.

In order to explore the limits of the traditional approach to this problem, the paper analyses Presenzini's grammar, first published in 1946, because it undertakes to outline some unifying principles and to explain the common roots of several phenomena and exceptions in French phonetics, graphematics and morphology.

These purposes are the ground of new grammar conceived for Italian universities (F. P. A. Madonia et A. Principato, Grammatica della lingua francese, Roma, Carocci, 2011).

\section{INDEX}

Keywords : French grammar, adult learning, grammar system, grammar exceptions, history of language

Mots-clés : grammaire française, apprentissage adulte, système grammatical, exceptions grammaticales, histoire de la langue 
AUTEUR

AURELIO PRINCIPATO

Université Roma Tre, Italie

aurelio.principato@uniroma3.it 